\title{
OLEOZON VERSUS MAJOR AUTOHEMOTHERAPY IN THE TREATMENT OF DRY SOCKET
}

\author{
Mosaad Abdaljawwad Abdalmawla Khalifah*
}

\begin{abstract}
Dry socket (DS) is a very common post-extraction complication. Pain is the most important clinical symptom in DS. Although many studies provided variant treatment methods for pain alleviation, there is still a wide controversy about their efficacy. Ozone achieved promising results in the treatment of variant conditions including DS due to its powerful oxidation and metabolism enhancement capacity, however, no enough data is available in the literature about its efficacy in DS treatment; particularly the comparative studies. In the current comparative study, forty patients with DS were randomly divided into two equal groups. In Group I, a brief saline irrigation of the extraction socket was followed by ozone major autohemotherapy (AHT) which was performed by withdrawing a venous blood to be ozonized; and then being reinfused into the vain. A cotton pellet impregnated with Oleozon was lightly packed in the socket after a brief saline irrigation in group II patients. Oleozon was more effective than AHT regarding the required total treatment period and the number of the required analgesic tablets. Oleozon might have an increased capacity of oxygen delivery to the ischemic tissues than AHT. This might provide an evidence for the ischemic etiology of the dry socket development.
\end{abstract}

KEY WORDS: Dry socket; Ozone; Autohemotherapy; Oleozon; Ischemia

\section{INTRODUCTION}

Dry socket (DS) is a common post-dental extraction complication ${ }^{[1]}$. Although it is considered a healing retardation, pain is the most important clinical consideration ${ }^{[2]}$. Multiple and variant treatment methods have been utilized to alleviate the pain but with a great controversy about their efficacies $^{[3-6]}$. Ozone is a very powerful healer and oxidant that has been clinically used in many different forms ${ }^{[7-9]}$. Ozone enhances oxygen delivery to all tissues especially the ischemic tissues, and therefore, improves the metabolism and activates the immune system. That accounts for its healing power ${ }^{[7,8,10,11]}$. Since Wolff has introduced ozone major autohemotherapy (AHT), it was used as a treatment method for a variety of conditions ${ }^{[12]}$ including $\mathrm{DS}^{[13]}$. Oleozon is an ozonized olive oil used to treat the dry socket ${ }^{[9,14,15]}$. No enough

* Lecturer of Oral \& Maxillofacial Surgery, Department of Oral \& Maxillofacial Surgery, Faculty of Dentistry, Kafr ElSheikh University, Kafr ElSheikh, Egypt. 
comparative studies in the literature to compare between different methods of ozone therapy in the treatment of $\mathrm{DS}^{[16]}$. The aim of the current study was to compare the efficacy of AHT and Oleozon as treatment methods DS.

\section{MATERIALS AND METHODS}

The current study was conducted on forty patients from the outpatient department of Oral and Maxillofacial Surgery department suffering dry socket. This study complied with the Declaration of Helsinki (revised in 1975), and the regional ethical review board. All patients provided informed consent. Inclusion criteria included simple extraction (forceps extraction) cases only, age range from 18 to 65 years, and to be otherwise healthy patients. Exclusion criteria included the existence of infected socket, any systemic or local disease affects healing, any bleeding disorder, perimenstrual extractions performed, pregnancy, lactation, the use of oral contraceptives, any hormonal disturbances or smoking. Patients were randomly divided into two equal groups; group I who undergone AHT procedures and group II who received oleozon. In group I patients, the socket was briefly irrigated with $2 \mathrm{ml}$ normal saline $(0.9 \%$ solution $)$ to remove any debris. Figure 1 shows a patient preoperatively. Major autohemotherapy was carried out by withdrawing $225 \mathrm{~mL}$ of patient's antecubital vein blood into a sterile glass bottle containing $25 \mathrm{~mL}$

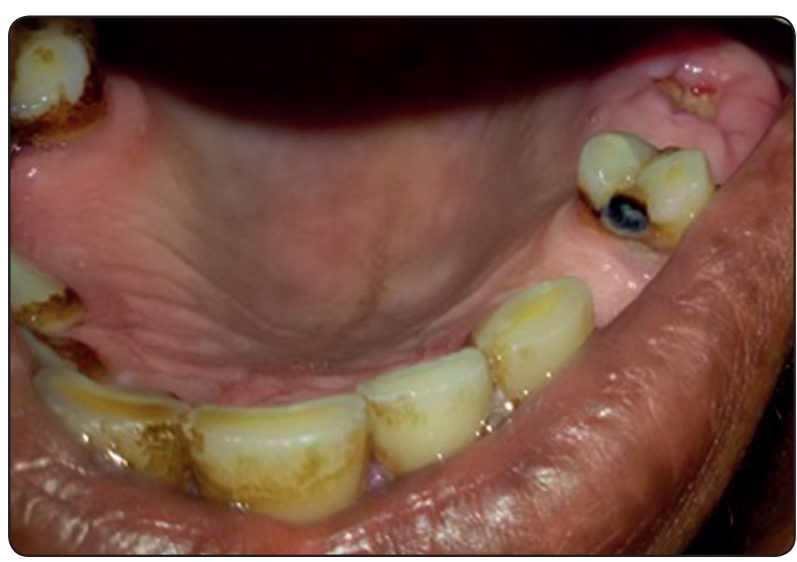

Fig. (1): Preoperative photograph for a case of major autohemotherapy. of 3.8\% Sodium Citrate solution (Al alamia gp, Cairo, Egypt) as an anticoagulant. The blood was then continuously mixed with $225 \mathrm{~mL}$ of OxygenOzone gas (Ozone concentration was $50 \mu \mathrm{g} / \mathrm{mL}$ gas; total dose of Ozone was equivalent to $11.25 \mathrm{mg}$ ) produced by an Ozonline 100 E generator (Medica srl, Bologna, Italy) (figure 2). Mixing was performed for at least 5 minutes with a concomitant gentle rotatory movement in order to avoid foaming. The process of ozonization is demonstrated in figure (2). Then blood is reinfused over 15-20 minutes (figure 3$)^{[12]}$. AHT was performed every three days

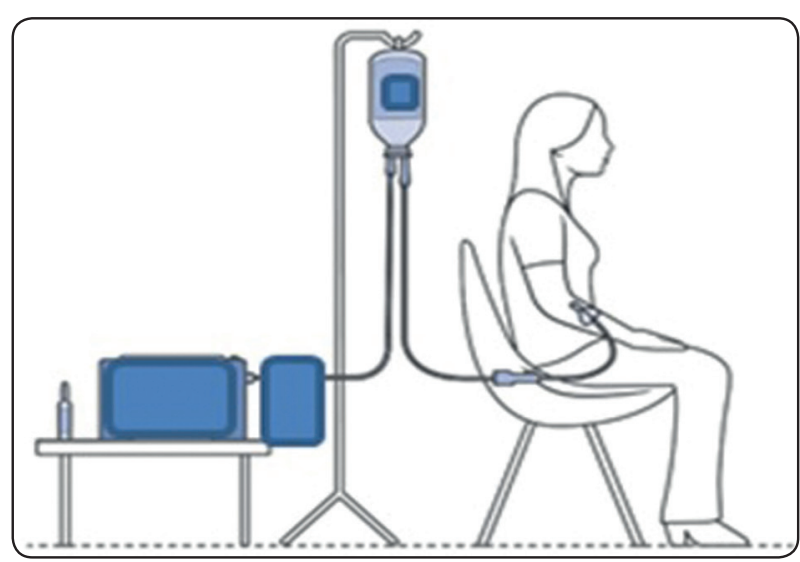

Fig. (2): Demonstration of the ozonization process.

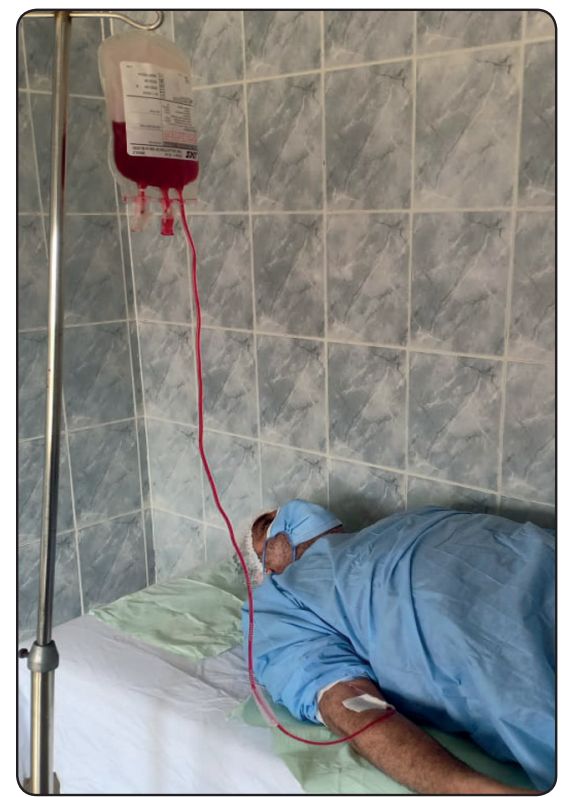

Fig. (3): Ozonizated blood reinfusion. 
if pain was not eliminated. Figure 4 shows a patient postoperatively. For group II, the socket was briefly irrigated with $2 \mathrm{ml}$ normal saline ( $0.9 \%$ solution) to remove any debris, followed by lightly packing with a cotton pellet impregnated with Oleozon (Alnour Advanced Technologies, $6^{\text {th }}$ October City, Cairo, Egypt). All patients were followed up daily until pain disappears. The treatment period was considered to be the total number of days for pain to disappear; and that was registered for every patient. The patient was asked to register how many tablets of diclofenac potassium $50 \mathrm{mg}$ analgesic (Cataflam 50, Novartis, Egypt) was used (if needed) daily to control pain. The total number of analgesic tablets taken by each patient during the total period of treatment was recorded. Data was collected and statistically analyzed using SPSS software (version 19, IBM Co, USA).

\section{RESULTS}

As table 1 show; the whole treatment period in group I patients was longer than that for the group II patients. Approximately, the period was doubled in group II patients. Also, the total number of analgesic tablets consumed by the patients in group I was approximately four folds that consumed by group II patients. The statistical difference with regards to the two tested parameters was significant.

TABLE 1

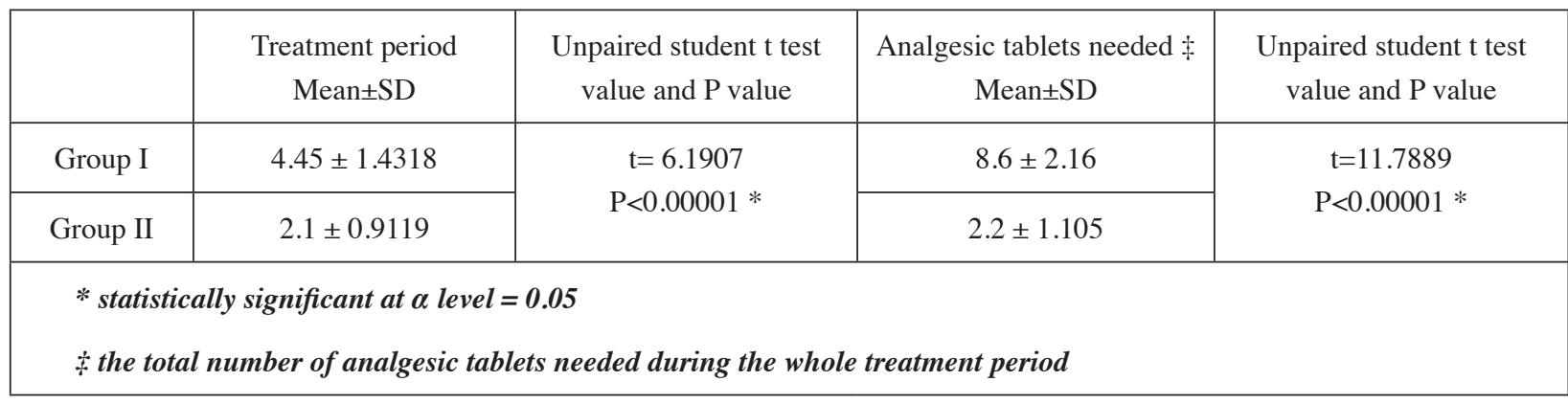

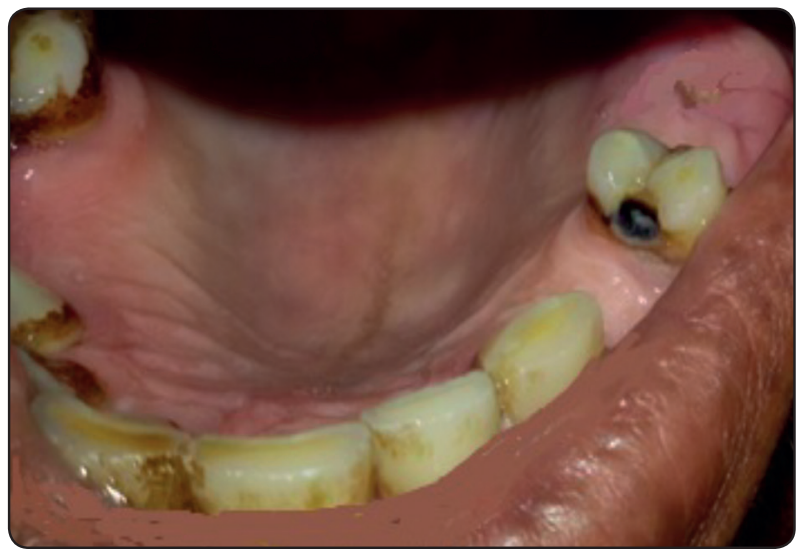

Fig. (6): Postoperative photograph for a case of major autohemotherapy at the fifth postoperative day.

\section{DISCUSSION}

Dry socket is a very common complication follows dental extraction ${ }^{[1]}$ with pain is the most important clinical issue but with a great controversy about the efficacy of its treatment methods ${ }^{[2-5]}$. Of these treatment modalities, ozone was utilized in a limited number of researches with promising results $^{[9,13,14]}$ due to capability to deliver oxygen to the ischemic tissues, and therefore, improves the metabolism and the healing and activates the immune system ${ }^{[7-11]}$. However, researches to compare the efficacy of different forms of ozone to treat DS is not enough ${ }^{[17]}$. Therefore, the current study was a trial to compare the efficacy of AHT and oleozon in the treatment of DS. 
In the current study, the inclusion and exclusion criteria were set to have as homogenous group as possible. Any patients with healing problem were not included. Patients had their teeth extractions during the perimenstrual period, pregnancy, lactation, or during the use of oral contraceptives were excluded from the study since these are risk factor for the development of DS and as a result might affect healing during the application of both treatment modalities of the current study. For the same reason, any patient with hormonal disturbances or a smoker patient was also excluded.

From the point of view of the current study, oleozon was more efficient that AHT; since oleozon had the capacity to decrease the total period required for pain alleviation by about $50 \%$ on comparing it with the AHT group. Also on the level of the required analgesics, AHT group mandated four folds the number of tablets required by oleozon group. It seems that local oxygen delivery to the socket bone is more powerful and more efficient than systemic oxygen delivery by the blood. Oxygen is an oxidant and enhances the metabolism which aid in removing the noxious stimuli affecting the nerve and causing pain. Lactic acid accumulation is also decreased by the reversal of the an-aerobic respiration process existing in the ischemic tissues. So, the added capacity of oxygen delivery by ozonization might have a role in reversing the ischemic state which might be present. The current study introduces the ischemic etiology for the dry socket development. Extraction forces applied to the bone lead to bone compression in a trial to expand the socket. When the buffering capacity of the blood supply is efficient to withstand these forces, there might be a very low risk for developing DS. Even though, if the buffering capacity cannot cope with these forces, there may be an interfere with the blood supply of the bone particularly the most peripheral portion which faces the root. As a result, a state of ischemia might issue leading to healing impairment, lactic acid formation, and inflammatory cytokines release which may ultimately cause blood clot disintegration, and pain. Oxygen as a sole treatment agent might have resulted in reversing this ischemic condition particularly when applied locally and with an intimate contact with the ischemic bone.

Although multiple reports recommended AHT as an effective modality to treat various conditions including DS, oleozon as a treatment modality for DS has the advantage of being much more easier for the operator and for the patient, more rapid, more available, and less costly rather than the AHT modality. Moreover, AHT requires more sophisticated equipment's than oleozon.

The results of the current study agree with those of other researches ${ }^{[14-16]}$. However, further studies are needed.

\section{CONCLUSION}

Dry socket is a very common post-extraction complication. Pain which is the most important clinical symptom had a wide controversy about the efficacy of its treatment modalities. Ozone achieved promising results in the treatment of variant conditions including DS, however, no enough data are available in the literature about its effect in DS treatment. In the current comparative study, oleozon was more effective than AHT, which might be due to the increased capacity of oxygen delivery of oleozon. This might provide an evidence for the ischemic etiology of the dry socket development.

The author declares no conflict of interest.

\section{REFERENCES}

1. A. Kolokythas, E. Olech, and M. Miloro, "Alveolar Osteitis: A Comprehensive Review of Concepts and Controversies,” Int. J. Dent., vol. 2010, pp. 1-10, 2010, doi: 10.1155/2010/249073.

2. M. Fazakerlev and E. Field, "Dry socket: a painful postextraction complication (a review)," Dent Updat., vol. 18, pp. 31-35, 1991, [Online]. Available: https://www. ncbi.nlm.nih.gov/pubmed/1936428. 
3. S. Faizel, S. Thomas, V. Yuvaraj, S. Prabhu, and G. Tripathi, "Comparision Between Neocone, Alvogyl and Zinc Oxide Eugenol Packing for the Treatment of Dry Socket: A Double Blind Randomised Control Trial," J. Maxillofac. Oral Surg., vol. 14, no. 2, pp. 312-320, 2014, doi: 10.1007/ s12663-014-0667-z.

4. R. Alexander, "Dental extraction wound management: a case against medicating postextraction sockets," J Oral Maxillofac Surg, vol. 58, no. 5, pp. 538-551, 2000, doi: http://dx.doi.org/10.1016/S0278-2391(00)90017-X.

5. I. R. Blum, "Contemporary views on dry socket (alveolar osteitis): a clinical appraisal of standardization, aetiopathogenesis and management: a critical review," Int J Oral Maxillofac Surg, vol. 31, pp. 309-317, 2002, [Online]. Available: http://dx.doi.org/10.1054/ijom.2002.0263.

6. M. Khalifah, "Curettage versus Zinc Oxide Eugenol as a Treatment Modality in Patients with Dry Socket," Egypt. Dent. J., vol. 67, no. 1, pp. 235-239, Jan. 2021, doi: 10.21608/edj.2020.48499.1322.

7. V. Bocci, I. Zanardi, and V. Travagli, "Ozone: A New Therapeutic Agent in Vascular Diseases," Am. J. Cardiovasc. Drugs, vol. 11, no. 2, pp. 73-82, Apr. 2011, doi: 10.2165/11539890-000000000-00000.

8. V. Bocci, Ozone A new medical drug, 2nd ed. Dordrecht. The Netherlands: Springer, 2011.

9. J. Martinez-Abreu, N. Guerra-Fonten, A. Blanco-Garcia, S. Naranjo-Rodriguez, E. Llanes-Llanes, and S. Menendez-Cepero, "Efficacy of OLEOZON® ${ }^{\circledR}$ compared to Alvogil in the treatment of alveolitis," J. Ozone Ther., vol. 1, no. 1, Dec. 2015, doi: 10.7203/jo3t.1.1.2015.12162.

10. V. Bocci, E. Borrelli, V. Travagli, and I. Zanardi, "The ozone paradox: Ozone is a strong oxidant as well as a medical drug," Med. Res. Rev., vol. 29, no. 4, pp. 646682, Jul. 2009, doi: 10.1002/med.20150.

11. V. Bocci, "Ozone as Janus: this controversial gas can be either toxic or medically useful.," Mediators Inflamm., vol. 13, no. 1, pp. 3-11, 2004, doi: 10.1080/0962935062000197083.

12. H. Wolff, "Die Behandlung peripherer Durchbutungsstorungen mit Ozon,” Erfahr Hk, vol. 23, pp. 181-184, 1974.

13. M. A. Khalifah, "Major autohemotherapy as a treatment modality for alveolar osteitis," J. Ozone Ther., vol. 2, no. 3, Dec. 2018, Accessed: Jun. 26, 2019. [Online]. Available: https://ojs.uv.es/index.php/JO3T/article/view/11292/pdf.

14. M. Khalifah, "A comparative study for the efficacy of different forms of ozone as a treatment for alveolar osteitis," Oral Surg., vol. 11, no. 3, pp. 195-199, Mar. 2018, doi: 10.1111/ors.12343.

15. J. A. Mazánek J, Hubálková H, Staňková H, Šmucler R, Linetskiy I, Seidler V, "Dry Socket aND The Length of Healing Depending on the Type of the Treatment Part Two: Treatment with Ozonated Water," Czech Dent. J., vol. 4, p. s. 96-101, 2016.

16. M. A. Khalifah, "Risk Factors, Etiology and Treatment Modalities for Localized Alveolar Ischemia (The So-called Alveolar Osteitis): A Comprehensive Critical Review," Ann. Oral Heal. Dent. Res., vol. 1, no. 2, p. R-7-R-14, 2018, doi: 10.21276/AOHDR.1917.

17. M.A.A. Khalifah, "Surgical Curettage as a Treatment Modality for Alveolar Osteitis: A Wide Controversy," Int. J. Clin. Oral Maxillofac. Surg., vol. 3, no. 5, pp. 26-29, 2018, doi: 10.11648/J.IJCOMS.20170305.11. 